\title{
Response by Authors to the Letter Regarding Article "Lidocaine and Pinacidil Added to Blood Versus Crystalloid Cardioplegic Solutions: Study in Isolated Hearts"
}

DOI: 10.21470/1678-9741-2019-0146

We appreciate the interest in our paper, entitled: "Lidocaine and Pinacidil Added to Blood Versus Crystalloid Cardioplegic Solutions: Study in Isolated Hearts" accepted on December 29th, 2017. Regarding the concerns raised in the letter to the editor ${ }^{[1]}$, we are thankful for the opportunity to reply to them. The study aimed to evaluate the effect about the association of very prominent drugs used as cardioplegic agents to achieve myocardial protection during elective cardiac arrest. Both pinacidil (only in experimental models!) and lidocaine are related to the maintenance of cell membrane polarization during ischemia, avoiding the intense ionic imbalance associated with ischemia-reperfusion injury ${ }^{[2]}$. A previous study to ours had shown that pinacidil associated with Custodiol solution showed superior effects compared to the original solution in an experimental model similar to the one we used ${ }^{[3]}$. Therefore, we investigated the possibility of the synergistic effect with lidocaine and pinacidil in the same solution not tested until that moment. In summary, the methods used in this experimental model evaluated left ventricular contractility, coronary resistance and myocardial injury. However, it is beyond our reach to perform a critical evaluation based on analyses in experimental model, animal species, preservation temperature, concentration and type of drugs different from those used by us. First, del Nido cardioplegia and Custodiol solution have an excellent spectrum of myocardial protection, therefore, a satisfactory scenario for the enrichment of their formulations with different drug combinations. Secondly, all contractility analyses were similarly applied to the three groups studied, e.g., control, del Nido and Custodiol, strictly following the recommendation methodology provided by the software company extensively used in the classic Langendorff apparatus model. Thirdly, myocardial pH control should be considered as an important complement for comparison between protective solutions, nonetheless, this study did not evaluate data which could infer that the differences found are related to $\mathrm{pH}$ or lactate content during ischemia. It is necessary to put the conditions of our experiments at the same level as the others to clarify the possible hypothesis discrepancies existence. In addition, the indicated reference ${ }^{[4]}$ for comparison to our study showed a high procaine concentration ( $<4 \mathrm{mmol} / \mathrm{L}$ ) applied on mongrel dog heart model[5], which is quite far from the model used by us.

Finally, we conclude under the conditions presented the modified cardioplegia del Nido-LP showed superior myocardial protection compared to modified Custodiol-LP. However, we endorse that the results showed do not have the same implication in the clinical usage until more evidence are found.

\section{Helison Pereira do Carmo' ${ }^{1}$, PhD}

(iD) https://orcid.org/0000-0003-0608-8640

'Universidade Estadual de Campinas, Unicamp, Campinas, São Paulo, Brazil.

\section{REFERENCES}

1. Preusse CJ. Comment on "Lidocaine and pinacidil added to blood versus crystalloid cardioplegic solutions: study in isolated hearts". Braz J Cardiovasc Surg. 2018;33(6):638-9. doi:10.21470/1678-9741-2018-0302.

2. Chambers DJ. Mechanisms and alternative methods of achieving cardiac arrest. Ann Thorac Surg. 2003;75(2):S661-6. doi:10.1016/S00034975(02)04688-X.

3. Ikizler M, Dernek S, Sevin B, Maxey TS, Kural T. Improved myocardial function with the addition of pinacidil to custadiol. Transplantation. 2002;74(12):1666-71. doi:10.1097/01.TP.0000038700.16562.7D.

4. Kresh JY, Nastala C, Bianchi PC, Goldman SM, Brockman SK. The relative buffering power of cardioplegic solutions. J Thorac Cardiovasc Surg. 1987;93(2):309-11.

5. Preusse CJ, Gebhard MM, Bretschneider HJ. Interstitial pH value in the myocardium as indicator of ischemic stress of cardioplegically arrested hearts. Basic Res Cardiol. 1982;77(4):372-87. doi:10.1007/BF02005338. 